\title{
Electron Microscope Study on the Lacrimal Gland of the Rat
}

\author{
By \\ Atsushi Ichikawa* and Yasuko Nakajima \\ From the Department of Anatomy, Faculty of Medicine, University \\ of Tokyo; Director: Prof. Dr. J. Nakai
}

(Received for publication, February 13, 1962)

\section{INTRODUCTION}

Several reports have been published on the fine structure of the lacrimal gland with the use of electron microscope. As far as the authors know, the first observation has been made on rabbit by Kobayashi ('58a) ${ }^{4}$, who has described that the acinar cell contains only one kind of secretory granules of less density and that the Golgi apparatus most intimately takes part in formation of secretory granule. Successively he has also reported on the human gland ('58b $)^{5}$. Following his works Obayashi ('59) ${ }^{10)}$ has discussed on the mechanism of secretory granule formation and recognized the presence of myoepithelial cells in acini and of secretory granules in the duct epithelia. Meanwhile Scott and Pease ('60) ${ }^{11}$ have mentioned in their study concerning the rat salivary and lacrimal glands that two kinds of secretory granules, zymogenic and mucous, are contained in the acinar cells and that myoepithelia were found to be limited to the intercalated duct of the lacrimal gland. More recently, Leeson ('60) ${ }^{9)}$ has reported on the presence of myoepithelial cells and their fine structures, in the rat exorbital lacrimal gland.

The authors consider that histology and cytology of the lacrimal gland contain some unsolved problems which should be re-examined, and would like to bring forward several questions as follows: (1) How many kinds of secretory granules are classified in acinar cells and/or duct epithelia? (2) Whether the endoplasmic reticulum or the Golgi apparatus and/or mitochondria do take part in granule formation? (3) With what mechanism are secretory granules released into the lumen? (4) What relationships are there among acinar cells, myoepithelia and nerve terminals?

This study aims at answering these questions.

* Present address; Department of Anatomy, Tohoku University School of Medicine, Sendai.

$$
\text { 市川厚, 中島泰子 }
$$




\section{MATERIALS AND METHODS}

Adult male rats were used in this investigation. Animals were divided into two groups, one of which consists of rats without any experimental treatment and those of the other group were injected subcutaneously with 0.4 to $0.6 \mathrm{cc}$ of $0.2 \%$ pilocarpine hydrochloride solution in order to stimulate the secretory function of the gland. The experimentally treated rats were sacrificed 10, 30, 90 minutes, 3 and 5 hours after injection, respectively.

The exorbital lacrimal glands were removed immediately after head blow and immersed in 1\% osmic acid solution buffered in the manner of Caulfield ('57). ${ }^{11}$ After initial immersion for a short time tissues were cut into small blocks, immersed again in the same fixative for one and a half to two hours, kept at $4^{\circ} \mathrm{C}$, washed briefly with distilled water and dehydrated in a series of increasing concentrations of ethyl alcohol. Each block was embedded with a mixture of $n$-butyl-and methylmethacrylate in the ratio of $8: 2$. Sections were cut with glass knives on a JUM-4 ultramicrotome. Electron micrographs were made with an HU-10 electron microscope at original magnifications of 2,000 to 10,000 diameters.

For light microscopy parts of tissues were fixed in Helly's solution and embedded in paraffine. Sections were stained with H.E., azan and PAS reaction with counterstain of iron-hematoxylin.

\section{RESULTS}

\section{Terminal portion}

General survey of the acinar cell:

In the cuboidal epithelium forming the terminal portion the round or oval nucleus stands somewhat lower than the center of cell body and well-developed lamellae of the rough-surfaced endoplasmic reticulum are found in the basal region. The lumen of the endoplasmic reticulum tends to be dilated and deformed in various ways. The reticula which lie in the peri- and supra-nuclear regions consist of round or ovoid cisternae. They are various in number and size, and scattered. In the supranuclear region well-developed Golgi apparatus in which the vacuolar elements are the most dominant, is found in any glandular cells. Mitochondria are scattered in cytoplasm and their features are rod-like in the basal and granular in the peri- and supra-nuclear regions. No distinct differences of the inner structures are found among them (Fig. 1). Mitochondria seem to gather toward the Golgi area, although there is no specific relationship of mitochondria to the other organellae. The supranuclear and apical regions are filled by large number of granules which are round or oval in shape. These granules are always surrounded with a thin membrane and their contents are homogeneous and various in density of transitional stages from rather less to somewhat high. The intercellular secretory canaliculi are well developed (Fig. 5). 
The myoepithelial cell:

Between acinar cells and the basement membrane myoepithelial cells are frequently observable. Generally the nucleus lies flattened in the center of cell body and organellae such as mitochondria and endoplasmic reticulum are mainly situated in the perinuclear region and poorly developed. Filamentous structures are present in the pale cytoplasm, especially in the peripheral. With such characteristics the cell is easily distinguishable from the acinar cell (Fig.s. 9 \& 10).

2. The duct system

The small duct neighbouring the terminal portion consists of a layer of flattened epithelia, of which the cytoplasmic matrix is characterized by higher density than that of the acinar cell. An ovoid nucleus lies in the center of the cell and a small number of dense granules are found in the apical and supranuilear regions (Fig. 3). Some granules which lie within the Golgi area are seen to be surrounded with a thin membrane in cross-section, meanwhile others lying beneath the surface plasma membrane seem to decrease in density and their limiting membrane is not obvious. The Golgi apparatus is large in size, although the other organellae such as endoplasmic reticulum and mitochondria are not so well-developed as those of the acinar cells (Fig. 4). Generally numerous microvilli are present on the free surface of plasma membrane and sometimes a small apocrine-like process is found (Fig. 8). No typical infolding of the plasma membrane is seen in the basal region of cell body, while intercellular boundaries are characterized by cellular interdigitation in complex arrangement (Fig. 7). Myoepithelial cells are also seen to lie within the basement membrane of the epithelia (Fig. 3).

A larger duct consists of two layers of epithelia, one of which lines the lumen of the duct is similar to that of the small duct. Basal cells contain the cytoplasmic matrix of less density, poorly developed organellae and show no infolding of the basal plasma membrane.

\section{Changes caused by pilocarpine treatment}

The remarkable changes are recognized in the case 30 minutes after injection, that is, decrease in number and fusion of secretory granules, increase in size of the Golgi complex and predominant development of the intra- and inter-cellular secretory canaliculi are seen in acinar cells (Fig. 5). Adhesion of the limiting membrane of secretory granule to the plasma membrane or deep depression of the plasma membrane into the cytoplasm are frequently found in the free surface of acinar cells (Fig. 6). In other cases, say, 1 and 3 hours after injection, the Golgi complex of acinar cells is distinctly developed, and various transitional forms from the Golgi vacuoles to the secretory granules or from the rough-surfaced vacuoles of the endoplasmic reticulum to the smooth-surfaced one are seen in the Golgi area (Fig. 2). 
No remarkable changes are found in the epithelial cells of duct system. Myoepithelial cells do not show any changes in their basic morphological features, too. However their cell bodjes sometimes penetrate deeply into the base of acinar cell or the intercellular space of glandular cells. In such cases, the intercellular space between the myoepithelial cell and the acinar or the duct epithelium is frequently dilated and small projections of glandular cell body which resemble microvilli are found there (Figs. $3 \& 10$ ).

\section{Nerve fibers}

Unmyelinated nerve fibers are often recognizable in the inter- and intralobular • connective tissues. Those in the former are approximately one or more micra in diameter and surrounded by the cytoplasm of Schwann cell, with or without mesaxon (Fig. 11), while in the latter their diameters are 0.2 to $0.4 \mu$ and also covered with Schwann cells (Fig. 12). However no finding that these fibers penetrate through the basement membrane of the glandular epithelia and directly attach to the acinar cells or myoepithelial cells, and/or no structure resembling the nerve terminal could be found in this investigation.

\section{DISCUSSION}

\section{On Secretion of the Glandular Cell in the Terminal Portion}

Numerous granules which lie in the supranuclear and apical regions of the acinar cell are surrounded by a thin membrane and their contents are homogeneous and various in degree of density, and the transitional forms are seen among them. Such granules decrease obviously in number after treatment with pilocarpine and are gradually reproduced in 3 to 5 hours. In cases of 1,3 and 5 hours after injection the Golgi complex of the acinar cell is well-developed and shows a transition of the Golgi vacuoles to such granules. This fact suggests that the secretory granule formation of the lacrimal acinar cells occurs within the Golgi complex, as well as in many glands, that is to say, the limiting membrane of granule is originated from the Golgi membranes. While granules migrate toward the apical region, the contents are matured increasing a little their density and are released into the lumen by typical mode of merocrine secretion. That is, after the complete fusion of the limiting membrane and the surface plasma membrane, an opening may occur at the site of membrane fusion and the granule contents may be discharged through the opening.

According to studies of Kobayashi ${ }^{4,5)}$ on rabbit and man and of Obayashi ${ }^{10)}$ on rabbit, only one kind of secretory granules are found in the lacrimal glandular cell and are arranged in a reticular form. Whereas Scott and Pease ${ }^{11)}$ claimed that the predominant acinar cells of the rat lacrimal gland are characterized by the presence of both light and dark granules which apparently represent an admixture of mucous and zymogenic secretory granules, respectively. However, no such 
dense granules as shown in their study have been ever found in this study. Moreover secretory granules consisting of only one kind are negatively stained by PAS reaction, although they considered that a part of secretory granules are mucous ones because the light type of granules are comparable in appearance and density with the formed mucous granules of the sublingual gland. It could be considered that these granules are water-rich and contain only a little salts or protein, and that have no mucous nature.

As regards the secretory granule formation, Kobayashi mentioned the close relationship between the Golgi apparatus and granules, while Obayashi described that the rough-surfaced endoplasmic reticulum may play an important role in granule formation. According to Scott and Pease, the light type of granules are seen to arise from terminal vacuolar enlargement of the Golgi membrane by a series of transitional stages. The results in this study also agreed with those of their observations.

As to how the secretory granules are released into the lumen, there is no report except for Obayashi's one. He found deep depressions of the surface plasma membrane in one of his cases 30 minutes after pilocarpine treatment and mentioned that this may suggest the release of secretory granules. Similar findings which are seen in this study have been found in other glands such as the pancreatic acinar cell (Watanabe et al., '59) ${ }^{12}$ ), the salivary glands (Ichikawa et al., ' 61$)^{3)}$ and so on. Ichikawa ('60)' identified them as representing a typical merocrin secretion and Kurosumi ('61)" classified such secretion as "The extrusion type IV". The predominant development of intracellular secretory canaliculi which occurs immediately after injection may arise from the fusion of secretory granules as a result of too intensive stimulation with pilocarpine.

\section{On the Duct System}

Dense secretory granules which lie in the apical region of small duct epithelia do not decrease distinctly in number after stimulation, that is their changes of the secretory cycle are not clear. It is, therefore, difficult to ascertain the site of granule formation. In the study of Scott and Pease, no direct relationship between the zymogen granules and the Golgi apparatus was demonstrable in the epithelial cells. However, the secretory granules which lie within the Golgi area are sometimes seen to be surrounded by a thin limiting membrane. This fact may suggest that the dense contents would be formed within the lumen of the Golgi membranes as well as in the pancreatic acinar cell, the anterior pituitary cell and so on. These granules seem to decrease their density a little beneath the surface plasma membrane and their limiting membranes are not clearly observable and moreover apocrine-like processes of cytoplasm are frequently seen near them. No finding suggesting release of these granules was demonstrable in this study, but they might lose their limiting membrane beneath the free-surface plasma membrane 
and be extruded in forming apocrine process, as Kurosumi et al. ('59) ${ }^{7)}$ have reported on the apocrine sweat glands.

It was difficult to classify the presence of typical infolding of the basal plasma membrane in the duct epithelia, although Scott and Pease have mentioned that there is some infolding of the basal plasma membrane in typical lacrimal secretory ducts.

\section{On the Myoepithelial Cell}

The existence of the myoepithelial cell in the terminal portion of the lacrimal gland has been proved by Kolossaw $(1898)^{6}$ ) with light microscope and by Oba yashi and Leeson with electron microscope. As mentioned above, the myoepithelial cell is characterized by the presence of myofilaments in the pale cytoplasm and is easily distinguishable from the acinar cells. It is surprising that Scott and Pease have described, "In the rat, myoepithelial cells were found to be limited to the intercalated duct of the lacrimal gland." It has been clarified in this study that myoepithelial cells are also present in larger duct of which epithelia contain secretory granules.

\section{On the Nerve Terminals}

In this study, unmyelinated nerve fibers are frequently found in the interstitial connective tissues among acini, but there is no evidence of direct attachment of nerve fibers to the acinar cells or myoepithelial cells through the basement membrane. In all cases examined, a part of cell body with pale cytoplasm and poorly developed organellae is frequently seen to penetrate deeply into the acinar cell or the intercellular spase of acini. It is not clear whether this part represents the nerve terminal or not, because it is difficult to distinguish it from the cross-section of myoepithelial process. It is questionable whether the structure which was represented as a naked nerve fiber by Scott and Pease is really nervous structure.

\section{SUMMARY}

The fine structure of the lacrimal gland and its changes of the secretory cycle have been studied by light and electron microscopy, on the rat.

1. The acinar cell contains only one kind of the secretory granule which is surrounded by a thin membrane originated from the Golgi membranes, and its contents are low in density, water-rich and not mucous. The secretory granule is extruded in a typical mode of merocrine secretion into the lumen.

2. Both in the small and larger ducts, dense secretory granules are found in their epithelial cells and seem to be formed within the Golgi complex. The mechanism of granule release is not clear but the apocrine-like processes are often found. No typical infolding of the basal plasma membrane is observable and intercellular boundaries are characterized by well-developed cellular interdigitation 
in complex arrangement.

3. The myoepithelial cells are present in the terminal portion and duct system which is lined with the epithelial cells containing secretory granules.

4. Unmyelinated nerve fibers are sometimes found in the interstitial connective tissue among acini, but there is no evidence of direct attachment of nerve fibers to the acinar cells or myoepithelial cells.

\section{Literature}

1) Caulfield, J.B., J. Biophys. Biochem. Cytol., 1957, 3, 827.

2) Ichikawa, A., Setai no Kagaku (Jap.), 1960, 11, 228.

3) Ichikawa, A. \& Ichikawa, M., Acta Anat. Nippon. (Jap.), 1961, 36, 196.

4) Kobayashi, M., Acta Soc. Ophthal. Jap. (Jap), 1958a, 62, 230.

5) Kobayashi, M., Acta Soc. Ophthal. Jap. (Jap.), 1958b, 62, 2208.

6) Kolossaw, A., Arch. mikrosk. Anat., 1898, 52, 1.

7) Kurosumi, K., Kitamura, T. \& Iijima, T., Arch. hist. jap., 1959, 16, 523.

8) Kurosumi, K., Internat. Rev. Cytol., 1961, 11, 1.

9) Leeson, C.R., Anat. Rec., 1960, 137, 45.

10) Obayashi, T., Acta. Soc. Ophthal. Jap. (Jap.), 1959, 63, 2631.

11) Scott, B.L. \& Pease, D.C., Am. J. Anat., 1960, 104, 115.

12) Watanabe, Y., Arakawa, K. \& Yamamoto, J., Proc. 15th Congr. Electron Microscopy Japan (Jap.), 1959, 79 .

Fig. 1. General survey of the acinus in the case 30 minutes after pilocarpine injection. Nucleus (N) lies somewhat lower in the center of cell body, and irregular-shaped lamellae of the endoplasmic reticulum (ER) are well-developed in the basal region. Mitochondria (m) are scattered in cytoplasm and various in shape. Secretory granules are seen in the supranuclear and apical regions and their contents are various in density of transitional stages from rather less (Sgl) to somewhat high (Sg2). The Golgi complex (G) and the intracellular secretory canaliculi (IC) are seen to be well-developed. 5,500 $\times$

Fig. 2. The Golgi complex of the acinar cell which is active in granule formation. In such a complex, various transitions from the Golgi vacuole (Gv) to the secretory granule $(\mathrm{Sg})$ or from the rough-surfaced vacuolar endoplasmic reticulum (ER) to the smooth-surfaced one are observed. $\mathrm{m}$; mitochondria, id; interdigitation of neighbouring cells. $37,000 \times$ 

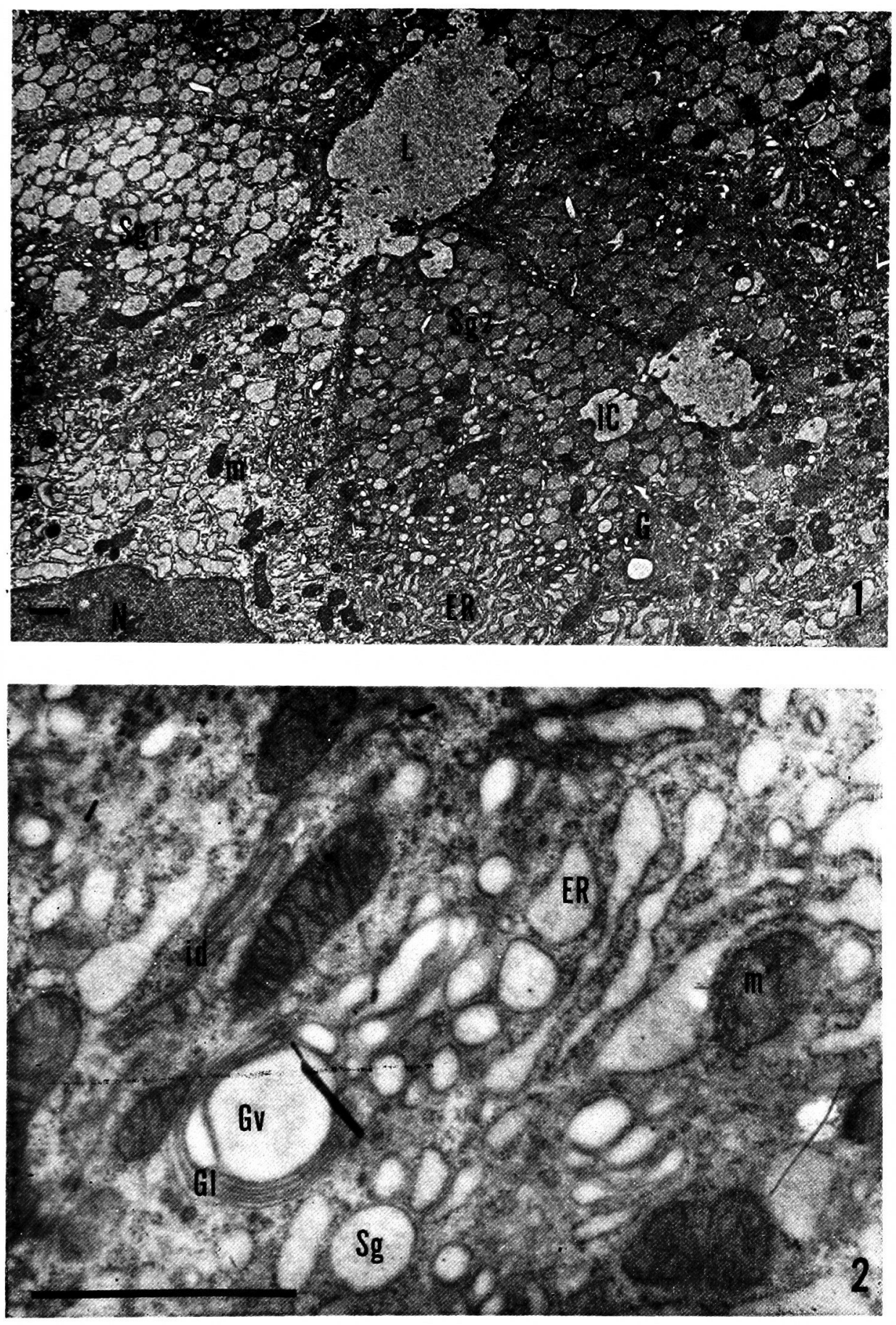
Fig. 3. A small duct neighbouring the terminal portion. Flattened epithelia lining the lumen (L) contain ovoid nuclei (En), dense cytoplasmic matrix and a small number of dense granules $(\mathrm{Sg})$ in the apical region. No typical infolding of the basal plasma membrane is found in the duct epithelia. In this case after stimulation, a predominant myoepithelium (M) and dilated intercellular space are seen between the duct epithelia and the basement membrane (bm). mv; microvilli. $7,800 \%$

Fig. 4. The apical region of the duct epithelia. Dense granules (Sg) within the welldeveloped Colgi complex ( $G$ ) seem to be surrounded by a thin membrane. Arrows show an aggregation of dense materials into small vesicles of the Golgi area. $m$; nitochondria. $23,600 x$ 

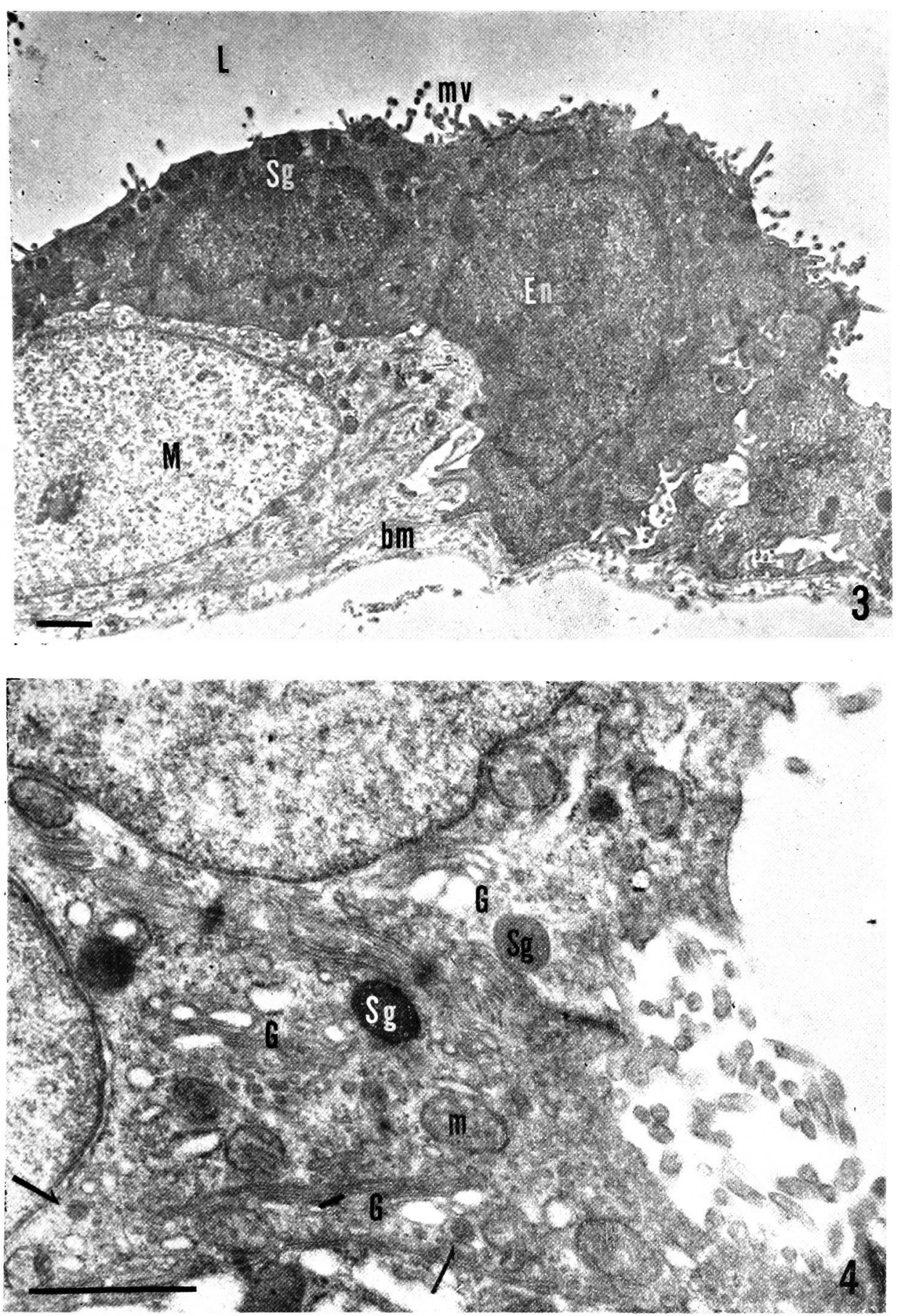
Figs. $5 \& 6$. The apical portion of acinar cells in the case 30 minutes after injection. Development of the intracellular secretory canaliculus (IC) which is probably originated from fusion of the secretory granules $(\mathrm{Sg})$ is prominent. Deep depressions of the surface plasma membrane (arrow) are frequently seen, and may suggest that the secretory granules extruded into the lumen $(\mathrm{L})$ through the opening of the plasma membrane which occurs in the site of fusion of both plasma membrane and the limiting membrane. In the middle of Fig. 6, a part of duct epithelium containing a small number of dense granules is seen. tb; terminal bar in the tangential section. Fig 5; 11,500 $\times$ Fig $6 ; 13,500 \times$

Fig. 7. The perinuclear regions of the duct epithelia. Intercellular boundaries of the duct epithelia are characterized by cellular interdigitation (id) in complex arrangement. $11,000 \times$

Fig. 8 The apical region of the duct epithelia in the case 30 minutes after injection. A small apocrine-like process (ap) is sometimes observed in the free surface. This may indicate that dense granules lose their limiting membrane beneath the free surface of epithelia and are released in forming apocrine process. $9,800 \times$ 

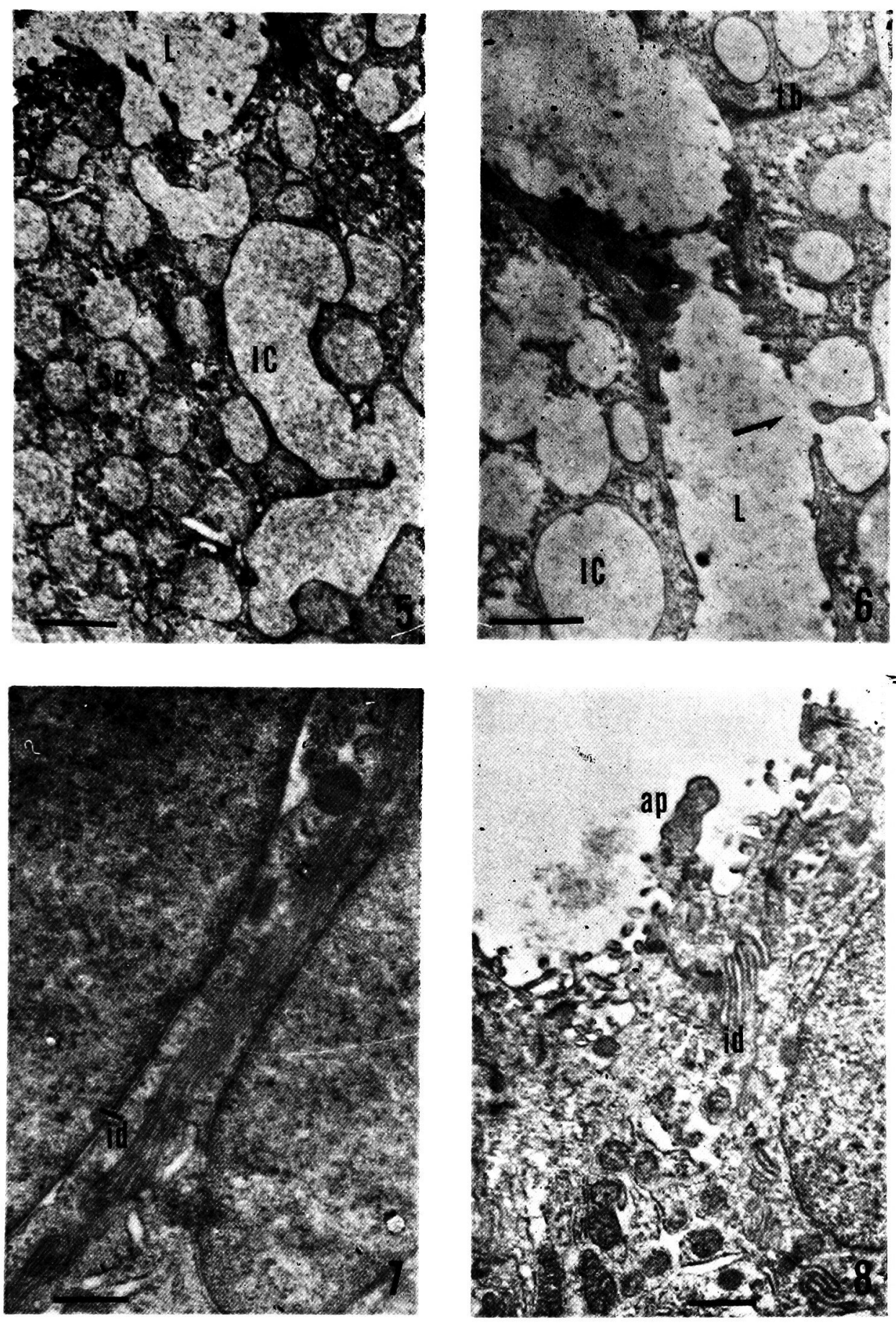
Fig. 9. Myoepithelial cell between the acinar cell (Ac) and the basement membrane (bm). Nucleus lies flattened in the center of cell body and organellae such as mitochondria (m) and endoplasmic reticulum are poorly developed. Filamentous structures (f) are present in the pale eytoplasm, espeeially in the peripheral region. $11,000 \mathrm{x}$

Fig. 10. The basal region of the terminal portion in the case 30 minutes after injection. The cell body of myoepithelium (M) sometimes penetrates deeply into the base of the acinar cells or the intercellular space of them. The intercellular spaces (is) between the myoepithelium and the acinar cell is frequently dilated and small projections resembling microvilli are seen there. $11,000 x$

Figs. $11 \& 12$, Unmyelinated nerve fibers (Nf) in tho intra- and interlobular connective tissues. Each axon $(\mathrm{Ax})$ is surrounded by the cvtomlusm of Schwann cell (Sn; its nucleus) with or without mesaxon, and its diameter is $0, \ldots 9.4$ in the former (fig. 11) and 1 or more micra in the latter (fig. 12). No finding ranbling the nerve terminals or attaching of these fibers to the a inar rells or myoe i lial cells (M) through the basement membrane could bc ivand. $11,000 \mathrm{w}$ 

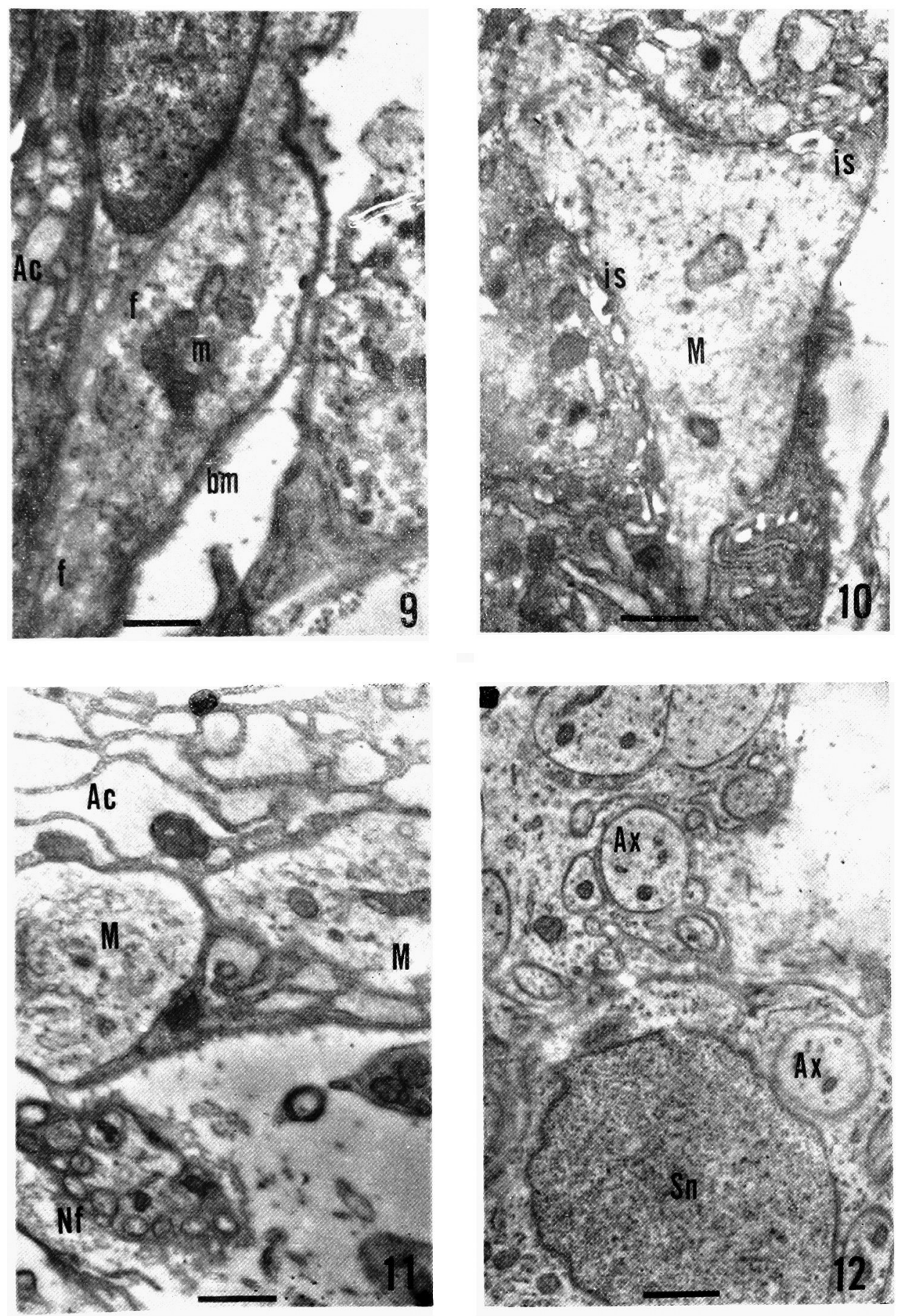